\title{
SONOGRAPHIC CORRELATION OF CAROTID INTIMA MEDIA THICKNESS AND HEPATIC ECHOTEXTURE WITH SERUM LIPID PROFILE
}

\author{
Sebastian Varghese ${ }^{1}$, Krishna Chaitanya Nunna ${ }^{2}$
}

1DNB, Department of Radiodiagnosis, Dr. Somervell Memorial CSI Medical College and Hospital, Karakonam, Kerala, India. ${ }_{2}^{2}$ MD, Dr. Somervell Memorial CSI Medical College and Hospital, Karakonam, Kerala, India.

\section{ABSTRACT}

\section{BACKGROUND}

Today ultrasound has emerged as a powerful diagnostic medical tool as it is cost effective, widely available and there is no risk of radiation. Driven by an increasing public awareness, as well as advances in treatment of dyslipidaemia, there has been a growing interest in the evaluation of carotid intima media for thickening and plaque and the assessment of liver echotexture for its fatty content by ultrasound over the last decade.

Aims and Objectives:

- To document the presence/ absence of carotid intima thickening in patients.

- To document the presence/ absence of plaque.

- To document the presence/ absence of diffuse alterations in hepatic echotexture for fatty changes.

- Sex and age prevalence of carotid intima media thickening and hepatic echotexture changes.

- Statistical correlation of carotid intima medial thickness and hepatic echotexture changes with serum lipid parameters, if any.

\section{MATERIALS AND METHODS}

This prospective observational study was performed in the Department of Radiodiagnosis and Imaging, Dr. Somervell Memorial CSI Medical College, Karakonam in the period between January - August 2017. A series of 241 consecutive adult patients who presented to our routine master health check-up were included in our study. Ultrasound analysis of the carotid arteries was performed with a high-resolution linear array transducer. The maximal intima media thickness and the presence of plaque were looked for on both sides. Using a curvilinear array transducer, the echogenicity of the liver parenchyma was evaluated. Serum total cholesterol, triglycerides, low density lipoprotein cholesterol (LDL-C) and high-density lipoprotein cholesterol (HDL-C) were estimated using enzymatic methods by a Hitachi 912 automated analyser.

\section{RESULTS}

- A total of 241 patients, who presented to our routine master health check-up underwent sonography and serum lipid analysis for the purpose of the study. Majority of patients in study population were males (62\%), while $38 \%$ were females. Of them, 150 were male and 90 were female. The mean age of the study sample was 55.1 years with a range of $19-83$ years. Maximum 148 (61.4\%) were in the age group of 41 - 60 years followed by $>60$ years' age group, which included 75 (31.2\%). Least number of patients $1(0.41 \%)$ were in the age group of $<20$ years. Carotid intima media thickening was found to be more common in males (42\%) than in females (29.6\%). In the $41-60$ years' age group it was $31.7 \%$ and $57.3 \%$ in the $>60$ years' age group.

- Presence of plaques also showed a gradual rise in prevalence with age, $23.5 \%$ in the 21 - 40 years' group, $33.1 \%$ in the $41-60$ years' groups and $65.3 \%$ in the $>60$ years' group.

- In patients with increased CIMT, the prevalence rate of abnormal lipid parameters range between $39 \%$ as with abnormal LDL-cholesterol and $45 \%$ as with abnormal HDL-cholesterol levels.

- $\quad$ Of the 241 patients who were evaluated for liver echotexture changes 130 (54\%) had diffusely increased echotexture, 111 $(46 \%)$ had normal and none $(0 \%)$ had decreased echotexture.

\section{CONCLUSION}

Twenty first century India is at a threshold of a cardiovascular atherosclerotic disease boom. Incidence of dyslipidaemia is on the rise. With the continuing rapid advances in ultrasound technology, sonography will continue to remain an effective diagnostic tool in the early assessment of carotid atherosclerotic disease and fatty liver disease.

\section{KEY WORDS}

Ultrasound; Plaque; Fatty Liver; Dyslipidaemia.

HOW TO CITE THIS ARTICLE: Varghese S, Nunna KC. Sonographic correlation of carotid intima media thickness and hepatic echotexture with serum lipid profile. J. Evolution Med. Dent. Sci. 2018;7(30):3380-3384, DOI: 10.14260/jemds/2018/763

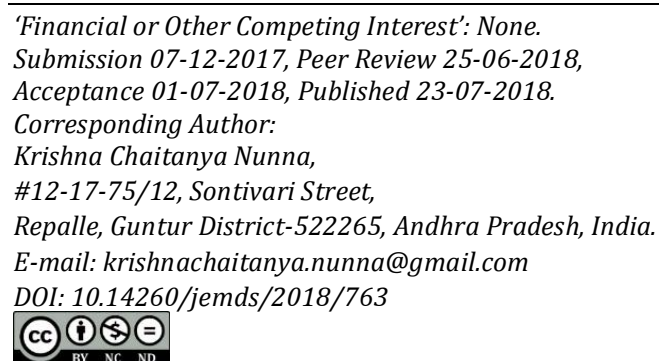

\section{BACKGROUND}

The applications of ultrasound have come a long way since the use of underwater sonar detection systems in World War I. Today, it has emerged as a powerful diagnostic medical tool with its cost effective, non-radiation and widely available advantages.

With the advent of high resolution ultrasound transducers, superficial structures get imaged with a high level of clarity and precision. 
The rapid spread of western lifestyles and a sedentary work culture have led to dyslipidaemia and its consequences becoming a major public health hazard.

Driven by an increasing public awareness as well as advances in treatment of dyslipidaemia, there has been a burgeoning interest over the last decade, in the evaluation of carotid intima media for thickening and plaque and the assessment of liver echotexture for its fatty content by ultrasound.

\section{Aims and Objectives}

- To document the presence/ absence of carotid intima thickening in adult patients.

- To document the presence/ absence of plaque.

- To document the presence/ absence of diffuse alterations in hepatic echotexture for fatty changes.

- Sex and age prevalence of carotid intima media thickening and hepatic echotexture changes.

- Statistical correlation of carotid intima medial thickness and hepatic echotexture changes with serum lipid parameters, if any.

- To help in deciding further course of management.

- To identify the high-risk category patients.

- To prove sonography will continue to remain an effective diagnostic tool in the early assessment of carotid atherosclerotic disease and fatty liver disease.

\section{MATERIALS AND METHODS}

This prospective observational study was performed in the Department of Radiodiagnosis and Imaging, Dr. Somervell Memorial CSI Medical College, Karakonam in the period between January - August 2017. A series of 241 consecutive adult patients who presented to our routine master health check-up were included in our study.

Ultrasound analysis of the carotid arteries were performed with a high-resolution linear array transducer. The maximal intima media thickness and the presence of plaque were looked for on both sides.

Using a curvilinear array transducer, the echogenicity of the liver parenchyma was evaluated. Spearman correlation was done for HE.

Serum total cholesterol, triglycerides, low density lipoprotein cholesterol (LDL-C) and high obesity lipoprotein cholesterol (HDL-C) were estimated using enzymatic methods by a Hitachi 912 Automated analyzer.

Study Design- A prospective observational study.

\section{Source of Data}

Patients referred to the Department of Radiodiagnosis, Dr. Somervell Memorial CSI Medical College, Karakonam for master health check-up.

Sample Size- The study comprised a total of 241 patients.

\section{Inclusion Criteria}

Patients who presented to our routine master health checkup.

\section{Exclusion Criteria}

Patients with focal liver lesions are excluded from the study.

\section{Statistical Analysis}

- All statistical analysis was done by using SPSS software trial version 20. Descriptive data was presented as percentages and tabulated.

- Chi-square test was done to assess the association among various categorical variables.

- $\quad$ The data were compared by the unpaired t-test between the groups with and without carotid plaques.

- Linear correlations between carotid IMT (dependent variable) with lipid parameters and clinical characteristics (independent variable) were evaluated by Spearman rank correlation as appropriate.

- For all statistical analysis, $\mathrm{p}<0.05$ was considered as statistically significant.

\section{RESULTS}

- A total of 241 patients, who presented to our routine master health check-up underwent sonography and serum lipid analysis for the purpose of the study. Majority of patients in study population were males (62\%), while $38 \%$ were females. Of them, 150 were males and 90 were females. There is male preponderance, male: female ratio being 1.65: 1 .

- The mean age of the study sample was 55.1 years with a range of 19 - 83 years. Maximum 148 (61.4\%) were in the age group of 41 - 60 years followed by $>60$ years' age group, which included 75 (31.2\%). Least number of patients $1(0.41 \%)$ were in the age group of $<20$ years.

- Carotid intima media thickening was found to be more common in males $(42 \%)$ than in females $(29.6 \%)$. None $(0 \%)$ of 18 patients in the $<40$ age groups had increased CIMT. In the 41 - 60 years' age group, it was $31.7 \%$ and $57.3 \%$ in the $>60$ years' age group.

- Presence of plaques also showed a gradual rise in prevalence with age, $23.5 \%$ in the 21 - 40 years' group, $33.1 \%$ in the $41-60$ years' groups and $65.3 \%$ in the $>60$ years' group.

- In patients with increased CIMT, the prevalence rate of abnormal lipid parameters range between $39 \%$ as with abnormal LDL-cholesterol and $45 \%$ as with abnormal HDL-cholesterol levels.

- $\quad$ For plaques, the prevalence rate varied between $39 \%$ with HDL-cholesterol and 49\% with total cholesterol.

- Of the 241 patients who were evaluated for liver echotexture changes, 130 (54\%) had diffusely increased echotexture, $111(46 \%)$ had normal and none $(0 \%)$ had decreased echotexture.

- In our study, we found 79 out of 150 males (52.6\%) and 51 out of 90 females $(56 \%)$ to have diffusely increased echogenicity suggestive of fatty liver.

- In our study, we found the lone 19-year-old patient $(<20$ years' group) to have fatty liver. The prevalence was $35.2 \%$ in the $>60$ years' group. 
Scatter Plot Diagrams
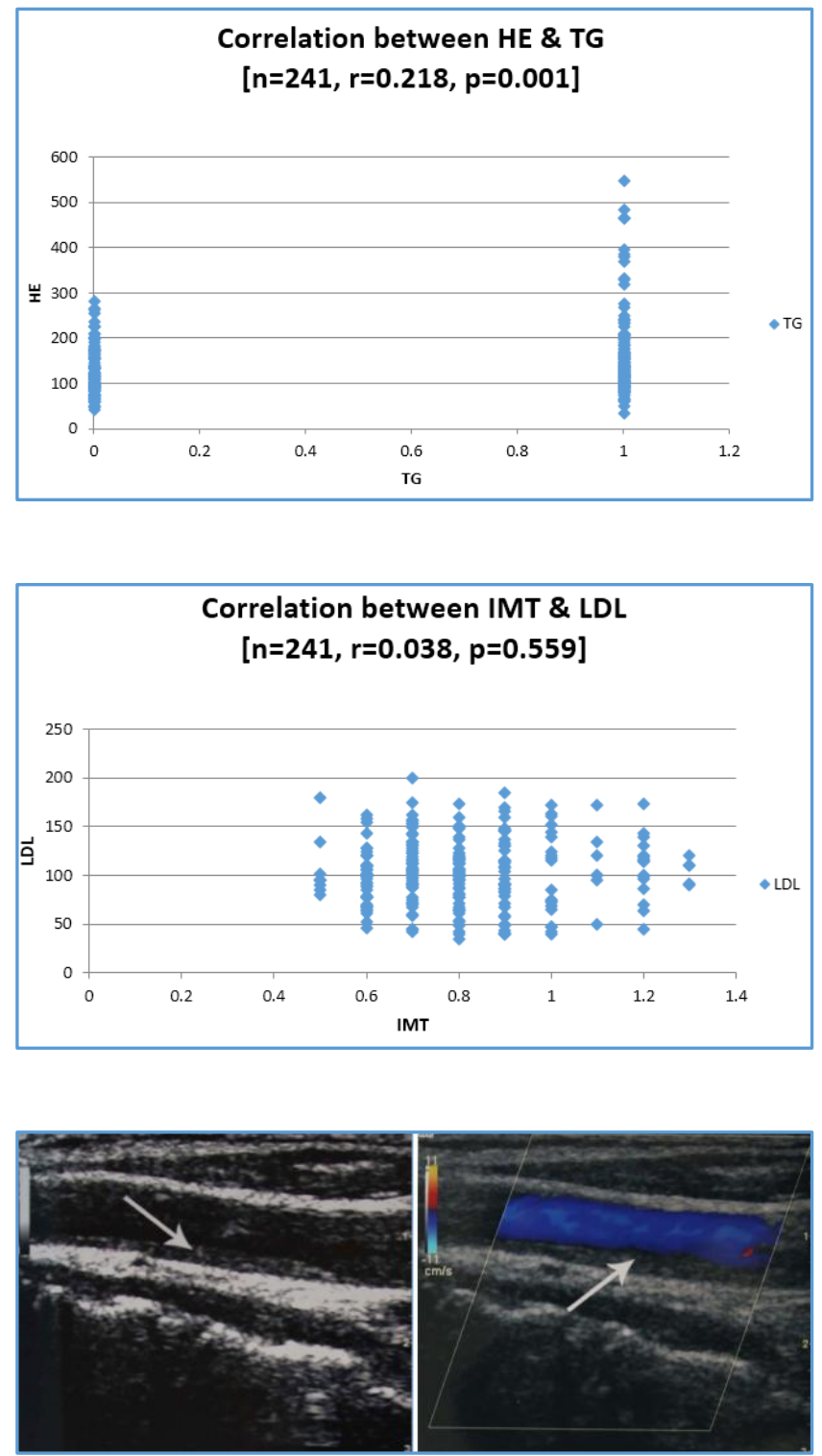

Long Segment Heterogeneous Hypoechoic Plaque causing Luminal Narrowing

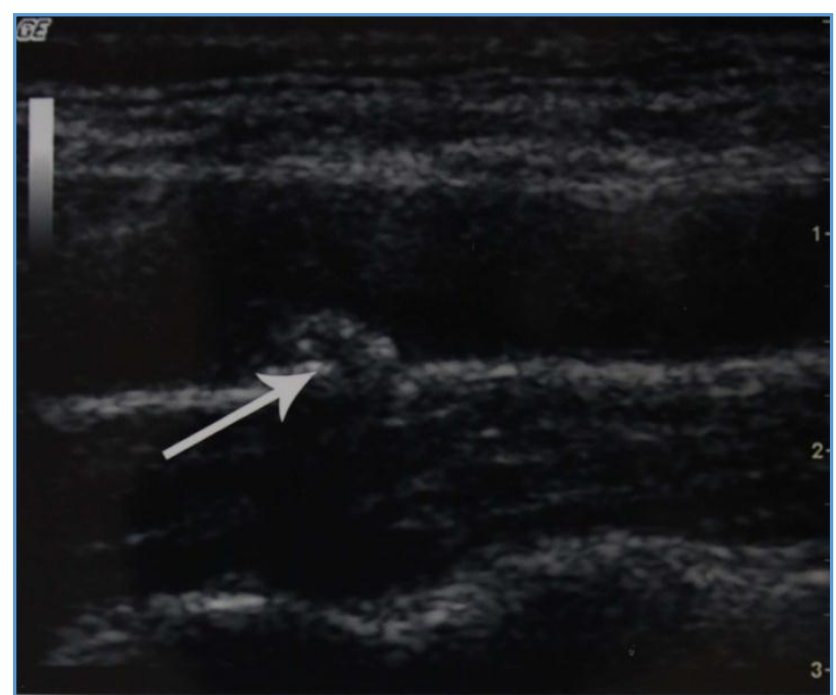

Heterogeneous partially Calcified Plaque

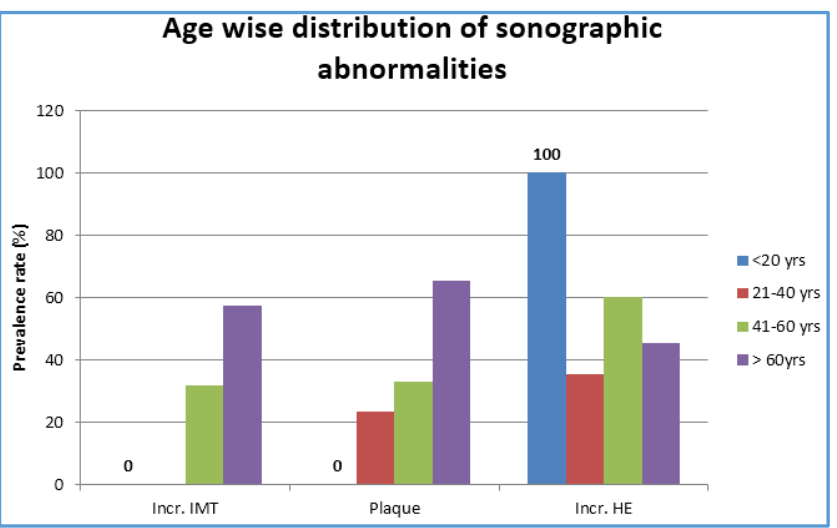

DISTRIBUTION OF DYSLIPIDEMIC PARAMETERS IN SONOGRAPHIC ABNORMALITIES.
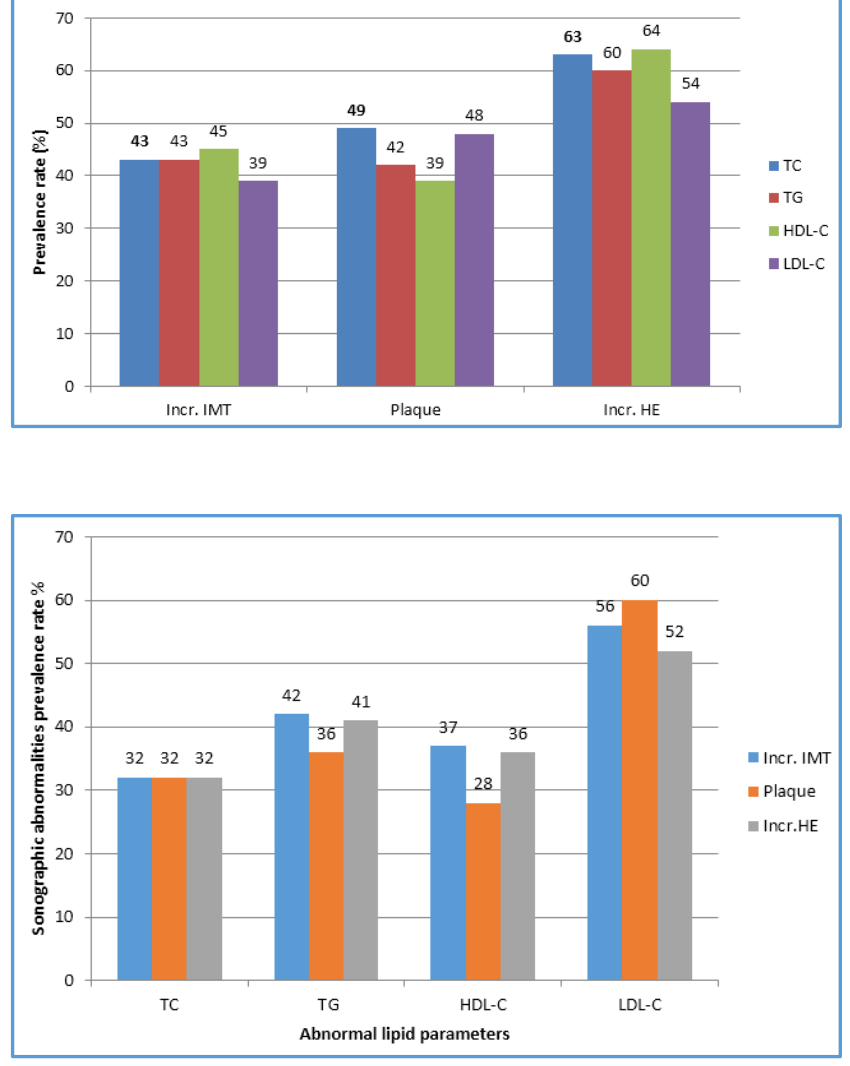

\section{DISCUSSION}

The purpose of our study was to determine using ultrasonography.

- The presence of carotid intima media thickening, plaque and fatty liver in our study population with quantification of sex/ age-wise prevalence, and

- Association with serum lipid parameters if any.

Our study population included 241 consecutive adult patients who presented to our routine medical health checkup with ages ranging from 19 - 83 years (mean 55.14 yrs.). Of them, 150 were males and 90 were females.

\section{Carotid Intima Media Changes}

Of 241 patients, 90 (37\%) had diffuse carotid intima media thickening. 
Carotid intima media thickening was found to be more common in males (42\%) than in females (29.6\%). 63 out of 150 males had increased CIMT as compared to 27 out of 91 females. These results were in accordance with the previous studies- Edinburgh Artery Study (Allan P et al) 1997, Stefan Rosfors et al (1998) and Baldasarre et al (2000).1,2,3

Plaques had an almost equal preponderance of $41.3 \%$ in males as compared to $43.9 \%$ in females.

Age has been a known risk factor for carotid intima media thickening. In our study, none $(0 \%)$ of 18 patients in the $<40$ age groups had increased CIMT. In the $41-60$ years' age group, it was $31.7 \%$ and $57.3 \%$ in the $>60$ years' age group. This gradual rise in prevalence of increased CIMT with age was in accordance with various studies including Lemne $\mathrm{C}$ et al and Jensen Urstal KJ et al.4,5 Presence of plaques also showed a gradual rise in prevalence with age, $23.5 \%$ in the $21-40$ years' group, $33.1 \%$ in the $41-60$ years' groups and $65.3 \%$ in the $>60$ years' group.

In patients with increased CIMT, we determined the prevalence rate of abnormal lipid parameters. We found them to range between $39 \%$ as with abnormal LDLcholesterol and $45 \%$ as with abnormal HDL-cholesterol levels. For plaques, the prevalence rate varied between 39\% with HDL-cholesterol and 49\% with total cholesterol.

Conversely, in dyslipidaemic patients we determined the prevalence rate of increased CIMT. It varied between 32\% with total cholesterol and 56\% with LDL-cholesterol. Plaques were present in $28 \%$ of the patients with abnormal HDL-cholesterol levels and $60 \%$ of patients with abnormal LDL-cholesterol. In short, carotid intima media thickening and plaques were found in $56-60 \%$ of patients with abnormal LDL-cholesterol levels.

The association of CIMT with serum lipid parameters has been a master of debate. Many studies have shown a significant association 6,7,8,9 with different lipid parameters, while a few have not. 10,11

While Baldasarre D et $\mathrm{al}^{3}$ in 2000 reported direct and significant correlations between carotid IMT and total cholesterol ( $\mathrm{r}=0.1, \mathrm{p}<0.05)$, LDL-cholesterol $(\mathrm{r}=0.13$, $\mathrm{p}<0.001)$ triglycerides $(\mathrm{r}=0.07, \mathrm{p}<0.05)$ and inversely with HDL-cholesterol, Zanchetti A et al (2001) found no relationship between IMT and any of the lipid parameters (Total cholesterol, LDL-cholesterol, HDL-cholesterol and triglycerides level) in a group of more than 500 patients with moderate hypercholesterolaemia and hypertension. ${ }^{12}$

In our study on obtaining the spearman correlation coefficient, all the lipid parameters positively but weakly correlated with CIMT, strongest being triglycerides and LDL-C $(n=0.038)$. However, no significant two tail 'p-value' was obtained for any of the lipid parameters.

\section{Hepatic Echotexture Changes}

Of the 241 patients who were evaluated for liver echotexture changes, 130 (54\%) had diffusely increased echotexture, $111(46 \%)$ had normal and none $(0 \%)$ had decreased echotexture.

Singh SP et al (2004) in a non-alcoholic population in coastal eastern India found the prevalence of fatty liver to be $24 \%$. It has been reported to be higher among those who consume large quantities ( $>60$ g per day) of alcohol (45\%), those with hyperlipidaemia $(50 \%)$ or obesity $(75 \%)$ and those with both obesity and high alcohol consumption
(95\%). ${ }^{13,14,15}$ Shen et al (2003) reported a higher prevalence in certain ethnic groups, e.g. upto $45 \%$ in the general population of Hispanic people. ${ }^{16}$

Our study population had a prevalence rate of $54 \%$ for fatty liver. Ours being an urban tertiary care multispeciality hospital, most of our study population consisted of upper middle and upper socio-economic status group patients. No patients with risk factors as mentioned in the studies above were excluded from our study, which could have contributed to the higher prevalence rate.

Most of the studies done on a non-alcoholic study population, for assessment of non-alcoholic fatty liver disease have reported the typical patient as a middle-aged women 81 - 85, while some have found a higher prevalence in males than in females.16,17,12,15

In our study, we found 79 out of 150 males (52.6\%) and 51 out of 90 females (56\%) to have diffusely increased echogenicity suggestive of fatty liver.

In our study, we found the lone 19-year-old patient $(<20$ years' group) to have fatty liver. The prevalence was $35.2 \%$ in the $>60$ years' group. Though in our study, we had not excluded any of the aetiological factors as mentioned in Table No. 1, the significantly increased prevalence in the 4160 years' group may be attributed to the lifestyle and food habit changes in our population over the last few decades.

In patients with sonological evidence of fatty liver, we determined the prevalence of dyslipidaemic parameters and found it varies from $54 \%$ with abnormal LDL-cholesterol to $64 \%$ with abnormal HDL-cholesterol. That is of the patients with sonological fatty liver, more than $50 \%$ were found to be dyslipidaemic.

When we determined the prevalence of sonological fatty liver in patients with abnormal lipid levels, we found it varied from $32 \%$ with abnormal total cholesterol level to a maximum of $52 \%$ with abnormal LDL-cholesterol.

In a study of 52 patients with hypercholesterolaemia, Assay N et al (2000) found $67 \%$ of the patients have normal ultrasound, whereas severe triglyceridaemia and mixed hyperlipidaemia were frequently associated with radiological evidence of fatty liver. ${ }^{17}$

In our study, we found that total cholesterol triglycerides and LDL cholesterol correlated positively and HDL-cholesterol inversely with sonological fatty liver.

While triglycerides showed statistically significant association ( $\mathrm{r}=0.218),(\mathrm{p}=0.001)$, which was in accordance with the Assay et al study, other lipid parameters showed no significant ' $p$ ' value.

\section{CONCLUSION}

Our prospective observational study with 241 adult patients revealed that carotid intima media thickness had a positive association with age and male gender, while fatty liver had a mild female preponderance.

In patients with carotid intima media thickening, the prevalence of dyslipidaemic parameters varied between 39 and $45 \%$, whereas with fatty liver disease it was significantly higher at 54 to $64 \%$.

Carotid intima media changes (thickening and plaques) were prevalent in upto $60 \%$ of the population with high LDL-cholesterol.

Carotid intima media thickness had a positive but weak relation with all the evaluated lipid parameters, while 
triglyceride levels showed a strong association with fatty liver.

Twenty first century India is at a threshold of cardiovascular atherosclerotic disease boom. Incidence of dyslipidaemia is on the rise. With the continuing rapid advances in ultrasound technology, sonography will continue to remain an effective diagnostic tool in the early assessment of carotid atherosclerotic disease and fatty liver disease.

\section{REFERENCES}

[1] Rosfors S. Hallerstam S. Jensen-Urstad $\mathrm{K}$, et al. Relationship between intima-media thickness in the common carotid artery and atherosclerosis in the carotid bifurcation. Stroke 1998;29(27):1378-82.

[2] Allan PL, Mowbray PI, Lee AJ, et al. Relationship between carotid intima-media thickness and symptomatic and asymptomatic peripheral arterial disease. The Edinburgh Artery Study. Stroke 1997;28(2):348-53.

[3] Baldassarre D, Amato M, Bondioli A, et al. Carotid artery intima media thickness measured by ultrasonography in normal clinical practice correlates well with atherosclerosis risk factors. Stroke 2000;31(10):2426-30.

[4] Lemne C, Jogestrand T, de Faire U. Carotid intimamedia thickness and plaque in borderline hypertension. Stroke 1995;26(1):34-9.

[5] Jensen-Urstad KJ, Reichard PG, Rosfors JS, et al. Early atherosclerosis is retarded by improved long-term blood glucose control in patients with IDDM. Diabetes 1996;45(9):1253-8.

[6] Skoglund C, Andersson, Tang R. LDL particle size distribution is associated with CIMT in healthy 50 years old men. Arterioscl Thromb and Vasc Biology: J of The American Heart Association 1999;19:2422-30.

[7] Wendelhag I, Wiklund 0, Wikstand J. Arterial wall thickness in Familial hypercholesterolemia. Ultrasound measurement of intima media thickness in common carotid artery. Atherosclerosis and thrombosis 1992;12(1):70-7.
[8] Boquist S, Ruotolo G, Tang R, et al. Alimentary lipemia, postprandial triglycerides-rich lipoproteins and common carotid intima-media thickness in healthy, middle aged men. Circulation 1999;100(7):723-8.

[9] Davis PH, Dawson JD, Riley WA, et al. Carotid intimamedial thickness is related to cardiovascular risk factors measured from childhood through middle age: The Muscatine study. Circulation 2001;104(23):28159.

[10] Saba PS, Roman MJ, Longhini C, et al. Carotid Intimamedial thickness and stiffness are not affected by hypercholesterolemia in uncomplicated essential hypertension. Arterio Thromb Vasac Biol 1999;19(11):2788-94.

[11] Weber F. Risk Factors for subclinical carotid atherosclerosis in healthy men. Neurology 2002;59(4):524-8.

[12] Luyckx FH, Desaive C, Thiry A, et al. Liver abnormalities in severely obese subjects: effect of drastic weight loss after gastroplasty. Int J Obes Relat Metab Disord 1998;22(3):222-6.

[13] Angulo P. Non-alcoholic fatty liver disease. N Eng J Med 2002;346(16):1221-31.

[14] Bellentani S, Saccoccio G, Masutti F, et al. Prevalence of and risk factors for hepatic steatosis in Northern Italy. Ann Intern Med 2000;132(2):112-7.

[15] Nomura H, Kashiwagi S, Hayashi J, et al. Prevalence of fatty liver in a general population of Okinawa, Japan. Jpn J Med 1988;27(2):142-9.

[16] Shen L, Fan JG, Shao Y, et al. Prevalence of nonalcoholic fatty liver among administrative officers in Shanghai: an epidemiological survey. World J Gastroenterol 2003;9(5):1106-10.

[17] Ruhl CE, Everhart JE. Determinants of the association of overweight with elevated serum alanine aminotransferase activity in the United States. Gastroenterology 2003;124(1):71-9. 